\title{
Positive Effects of Workplace Meditation Training and Practice
}

\author{
Associate Prof Leila Karimi ${ }^{1}$, Prof Stephen P Kent ${ }^{1}$, Prof Sandra G. Leggat ${ }^{1}$, Dr Jiri Rada ${ }^{1} \&$ Alison Angleton ${ }^{1}$ \\ ${ }^{1}$ School of Psychology and Public Health, La Trobe University, Melbourne, Australia \\ Correspondence: Associate Prof Leila Karimi, School of Psychology and Public Health, La Trobe University, \\ Plenty Road, Melbourne VIC 3086, Australia. E-mail: 1.karimi@latrobe.edu.au
}

Received: December 6, 2018

Accepted: January 2, 2019

Online Published: January 8, 2019

doi:10.5539/ijps.v11n1p15

URL: https://doi.org/10.5539/ijps.v11n1p15

\section{Ethical Standards}

The project was approved by the La Trobe University College Human Ethics Sub-Committee (CHESC) (approval number: S15-29).

\section{Informed Consent}

Written informed consent was obtained from all individual participants prior to their inclusion in the study.

\section{Conflict of Interest}

The authors declare that they have no conflict of interest.

\begin{abstract}
There is evidence that meditation is a powerful organisational tool for enhancing employee effectiveness, wellbeing, and job satisfaction; however, experimental studies on the effects of meditation on other organisational factors such as presenteeism and emotional intelligence are limited. This study investigated the impact of meditation on mindfulness, emotional intelligence, job satisfaction, and job stress-related presenteeism in an Australian workplace. Participants learned and practised an 'Auto Transcending Meditation Technique' (ATMT) at their workplace. The study used the switching replications experimental design, comparing an intervention group with a control group. Quantitative data analysis used descriptive statistics and repeated measures to compare the mean pre-post intervention differences. Thematic analysis was completed on qualitative data gathered in focus groups and from the training evaluation. As a consequence of ATMT, participants showed significant improvements in their levels of mindfulness and emotional intelligence. Thematic analysis indicated that participants felt the meditation training and practice led to positive personal changes. In addition, the results showed that higher mindfulness buffers the effect of stress-related presenteeism on participants' mental and physical health. Our results demonstrate that meditation training and practice enhances mindfulness and emotional intelligence, with benefits for employees' physical and mental health. Workplace meditation should be considered in health promoting work settings.
\end{abstract}

Keywords: mindfulness, meditation, emotional intelligence, presenteeism, stress, wellbeing

\section{Introduction}

Meditation is a popular tool to enhance employee effectiveness, health, and job satisfaction in many organisations (Goodson, 2013). Different cultures have practised meditation in various forms to connect the mind, body and spirit for hundreds of years. Traditionally, meditation focuses on improving relaxation, creating internal energy and positive feelings such as love, patience, and forgiveness (University of Wisconsin-Madison, 2008). Meditation techniques differ, but commonly include controlled breathing, repetitive phrases (mantra), and visualisations; all forms of meditation share mindfulness and self-awareness skills (Charoensukmongkola, 2014). A recent systematic review on the effects of mindfulness on stress measures and anxiety showed that meditation is associated with decreases in heart rate, blood pressure and cortisol levels, especially in those with more practice and strong focus on breathing (Pascoe \& Crewther, 2016).

According to the Global Burden of Disease Study 2010, anxiety is a leading source of adult disability worldwide (Sharma \& Rush, 2014) and workplace stress is a major contributor to anxiety (Colligan \& Higgins, 2006). Meditation has been promoted as effective management for stress and anxiety by various government and health 
organisations (healthdirect, 2016; National Institutes of Health, 2016). Indeed, meta-analyses and systematic reviews, show that various meditative practices are beneficial in reducing anxiety, stress, job tension, insomnia and fatigue, and can improve mood as well as general health in various populations (Alexander et al., 1993; Goyal et al., 2014; Khoury, Sharma, Rush, \& Fournier, 2015; Kulkarni, Janakiram, \& Kumar, 2009; Sharma \& Rush, 2014). Shonin et al. (2014) conducted a randomised controlled trial to explore the effects of meditation on work-related well-being and job performance. They found that after eight weeks of meditation training, participants showed lower levels of work-related stress and psychological distress, and higher levels of job satisfaction and performance. Meditation has also been found to improve work engagement, subjective job performance, and job satisfaction (Shiba, Nishimoto, Sugimoto, \& Ishikawa, 2015). A recent randomised controlled trial on adults with Generalized Anxiety Disorder (GAD) found associations between mindfulness practices and less work loss and fewer mental health professional visits (Hoge et al., 2017).

While there is evidence linking meditation with reduced stress and anxiety in the workplace, stress-related presenteeism has not received much attention. Stress-related presenteeism occurs where 'employees are physically present at work, but their cognitive energy is not devoted to their work' (Gilbreath \& Karimi, $2012 \mathrm{p}$. 2). It is surprising that this presenteeism has not received greater attention, given that it costs the Australian economy approximately $\$ 14$ billion a year (Medibank Private, 2008). In addition to the health risks to the employees, the net result for employers is substantial loss of worker productivity.

Intrapersonal resources and capacities have an impact on employees' reactions to their workplace demands (Laschinger. et al., 2016). Emotional Intelligence is an important intrapersonal resource that can buffer the impact of workplace stress (Karimi, Cheng, Bartram, Leggat, \& Sarkeshik, 2015; Karimi, Leggat, Donohue, Farrell, \& Couper, 2014). Emotion Regulation Theory (Baumeister \& Heatherton, 1996), one of the most used theories for the study of emotion at work, helps explain the empirical evidence. Emotion regulation refers to 'the processes by which individuals influence which emotions they have, when they have them, and how they experience and express these emotions' (Gross, 1998 p. 275).

Studies show that meditation is positively associated with EI (Chiesa, Calati, \& Serretti, 2011). Individuals who completed meditation training showed significant improvements in EI and stress reduction compared with the control group (Chu, 2010). Mindfulness skills and EI were correlated significantly with positive affect, life satisfaction, and lower negative affect, with EI mediating the relationship between mindfulness and these outcomes (Schutte \& Malouff, 2011). A recent study replicated the finding that mindfulness meditation practice enhanced EI and was directly related to decreased perceived stress (Charoensukmongkola, 2014).

Building on these findings we assessed the ability of a workplace meditation program to impact mindfulness, EI, job-satisfaction, and stress-related presenteeism. We used a mixed-methods experimental approach to answer the following research questions:

1. Does meditation training and participation enhance skills in mindfulness and emotional intelligence?

2. Does workplace meditation training and participation lead to increased job-satisfaction and decreased stress-related presenteeism?

Long-term exposure to stressful workplace situations can lead to mental and physical health problems. However, transactional models of stress, which assess the way individuals react to stressful conditions, suggest individual factors such as self-awareness, coping strategies, and emotion regulation may moderate the experience of stress on health (Slaski \& Cartwright, 2002). A few studies have provided support for the role of mindfulness and EI in reducing the health effects of stress (Charoensukmongkola, 2014; Schutte \& Malouff, 2011). However, there is little literature on the relationship between mindfulness and stress-related presenteeism. The role of self-awareness and mindfulness in stress-related presenteeism and the physical-mental health relationship remains to be fully investigated, leading to our third research question:

1. Does mindfulness moderate the effects of stress-related presenteeism on perceived health?

\section{Methods}

\subsection{Participants}

Study participants were clerical and administrative personnel at the corporate headquarters of a leading Australian wellness company, who learned and practised meditation at their workplace. Participation was voluntary; there were neither incentives nor disadvantages, penalties, or adverse consequences for not participating or for withdrawing before the study was completed. Participants were identified by the researchers only by assigned code numbers. The researchers were interested solely in aggregated relationships among the 
key concepts. Trainees received a letter of invitation that included a participant information statement, the consent form, and a link to the online survey.

\subsection{Meditation Training Intervention}

The training and data collection for the project started at baseline and continued at two-monthly intervals for four months. The meditation program was delivered by a certified meditation teacher and the participants were trained in 'Auto Transcending Meditation Technique' (ATMT). The technique was taught over four 120-minute consecutive sessions at the workplace each morning before work. After the four days of training, the participants meditated at the workplace daily in a group for two 20-minute sessions; at $8 \mathrm{am}$ and $3 \mathrm{pm}$.

ATMT is an effortless technique that requires no focus, concentration, or control of the mind (Travis et al., 2010). Automaticity can be achieved through extensive rehearsal which leads to 'effortless' concentration (Lutz, Slagter, Dunne, \& Davidson, 2008), utilising the repetition of a mantra. The aim of transcending refers to moving the mind from the gross level of thinking to experience ever subtler levels of thought and awareness.

\subsection{Timeline and Study Design}

The study used the switching replications experimental design, which has strong internal validity and is highly ethical because all participants eventually receive the intervention. Group $1(n=35)$ received the training at the outset and meditated for two months (i.e. no waiting period) and Group $2(\mathrm{n}=34)$ acted as the control group with no training or meditation practice for the first two months (i.e. delayed training). After two months, Group 2 also received the training (delayed training group) and commenced meditation, while Group 1 continued with two more months of meditation practises.

\subsection{Measures}

Freiburg Mindfulness Inventory (FMI). The FMI is a valid and reliable questionnaire to assess mindfulness' (Walach, Buchheld, Buttenmuller, Kleinknecht, \& Schmidt, 2006). The FMI questions focus on personal experiences. One item, 'I am impatient with myself and with others' needed to be reverse coded. The responses were Likert-based, ranging from 1 to 4 , and representing 'rarely true' to 'almost always true' answers. An example question was 'I am open to the experience of the present moment'. A single score was calculated to measure mindfulness; higher scores demonstrate higher levels of mindfulness. The reliability assessment for this sample indicated high reliability for this scale (time $1 \alpha=.89$, time $2 \alpha=.90$, time $3 \alpha=.88$ )

Emotional Quotient Inventory (EQ-i $2.0 \AA$ ). The EQ-i $2.0 \AA$ is a scientifically validated emotional intelligence measure. It examines an individual's social and emotional strengths and weaknesses (Statistical Solutions, 2015). Respondents self-report on their life and workplace performance in a general score in self-perception, self-expression, interpersonal skills, decision-making, and stress management. The inventory measures 15 key areas of emotional skills that contribute to proficiency in complex activities such as conflict resolution and planning. The reliability of the scale was reported as high in all previous studies. Due to copyright restriction, it was not feasible to calculate reliability of the scale for this sample.

Job Satisfaction Scale. Job satisfaction refers to how content an individual is with their job, or whether they like the job or individual aspects or facets of the job. It was measured using the six questions of the Job Satisfaction Scale developed by Seashore et al.(1982). The responses range from "strongly disagree" to "strongly agree"; a higher score indicates higher job satisfaction. The reliability of the scale in this sample was excellent at all three time points (time $1 \alpha=.92$, time $2 \alpha=.95$, time $3 \alpha=.92$ ).

Job Stress-Related Presenteeism. Presenteeism was measured by a six-item scale developed by Gilbreath and Frew (2008). The scale anchors are "all the time" (5) and "never" (1). The higher scores in this scale indicates high level of perceived stress-related presenteeism. Example questions for this scale are "I'm unable to concentrate on my job because of work-related stress' and 'Work stress distracts my attention away from my job tasks'. The internal consistency of the scale scales were excellent at all three time points demonstrating the reliability of the scale in this sample (time $1 \alpha=.88$, time $2 \alpha=.92$, time $3 \alpha=.92$ ).

\section{Short-Form 12 Health Survey (SF-12).}

The Short Form 12 item health questionnaire (Ware, Kosinski, \& Keller, 1996) evaluates aspects of an individual's self-reported physical and mental health using 12 Likert scale items. The SF-12 has eight subscales that collapse into two composite scales of mental health and physical health, where lower scores indicate better health. The two composite scales have both successfully predicted poor health outcomes from large sample studies (Herrman et al., 2002; Ikin et al., 2004; Taylor et al., 2000). The internal consistency of both physical 
health (time $1 \alpha=.70$, time $2 \alpha=.70$, time $3 \alpha=.81$ ) and mental health (time $1 \alpha=.82$, time $2 \alpha=.84$, time $3 \alpha$ $=.75$ ) scales were acceptable at all three time points demonstrating the reliability of the scale in this sample.

\subsection{Data Collection and Analysis}

The baseline measures were collected using an online survey at the beginning of the study (Time 1). Follow-up measures were collected at two-monthly intervals for four months, Time 2 and Time 3 respectively. The online survey was conducted using Qualtrics Survey Software (Qualtrics, Provo, UT). Quantitative data analysis was conducted using descriptive statistics and repeated measures to compare the mean pre-post intervention differences. The data were analysed using SPSS (v 21). Data were screened for outliers, errors, or missing data. Because of the longitudinal design of the study, more data were missing towards the end. Only participants who continued with the study to the end were included in the data analysis. Analysis of the patterns of missing data indicated that data were missing at random, suggesting that they can be fully accounted for by variables where there is complete information. Six percent of values missing and were replaced with mean substitution (Tabachnick \& Fidell, 2013).

Three focus groups were held after the final training sessions to explore the satisfaction of participants with the meditation training and practice. The groups explored what was helpful about the training, what aspects of the training could be improved, what specific benefits the participants experienced, and how the participants will apply the learning to their job role. To complement the quantitative data, the data gathered in the focus groups and from the training evaluation was subjected to thematic analysis, independently completed by two researchers. The process involved familiarisation with the data and creation of preliminary codes to produce a list of items with a recurring pattern, searching for and refining overarching themes, and substantiating each theme based on the data.

\section{Results}

\subsection{Demographic Characteristics}

The majority of the 35 participants were female (68\%), worked full-time $(95 \%)$, and possessed a tertiary qualification (82.5\%). As summarised in Figure 1, the response rate to the online surveys for Group 1 was 31/35 and 28/35 at two and four months respectively; for Group 2, the response rate was 29/34 and 12/34 for the same periods. The major reasons for the decrease in response rates included participants ceasing meditation, leaving the company, and being away during the data collection period.

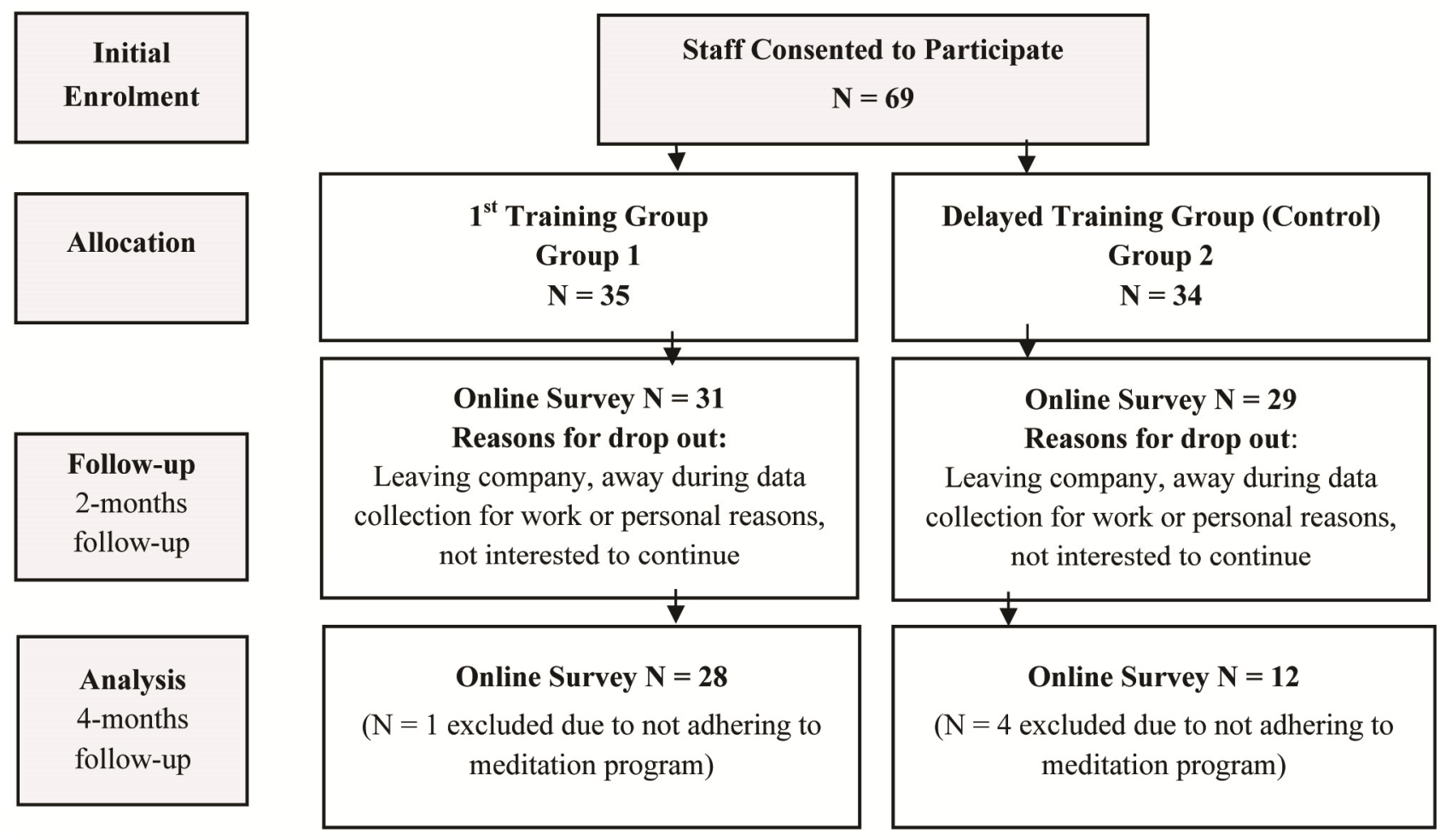

Figure 1. Participation data 


\subsection{Quantitative Analysis}

Mindfulness skills. A 2 (Group) x 3 (Time) repeated measures ANOVA was conducted to compare the scores on the FMI over time and between groups. The main effect for group was not significant; $F(1,38)=1.04, p=0.31$ with a small effect size of $\eta 2=.027$, suggesting that each group's overall mean from the three phases did not differ (Figure 2). The main effect for time revealed that both training groups improved their mindfulness skills across the three phases of the study; $F(2,38)=6.38, p=0.003$, with a larger effect size of $\eta 2=.140$. We do not report the interaction effect (comparison of group trends across phases), as the control group, unlike a traditional control group, is expected to increase the effect when it begins the meditation phase.

Emotional intelligence. The main effect between groups was not significant; $F(2,38)=2.48, p=0.12$, with a small effect size of $\eta 2=.061$ (Figure 3). However, the results indicate that the training groups showed significant improvement for emotional intelligence skills over time; $F(2,38)=11.91, p=0.001$, with a large effect size of $\eta 2=.239$.

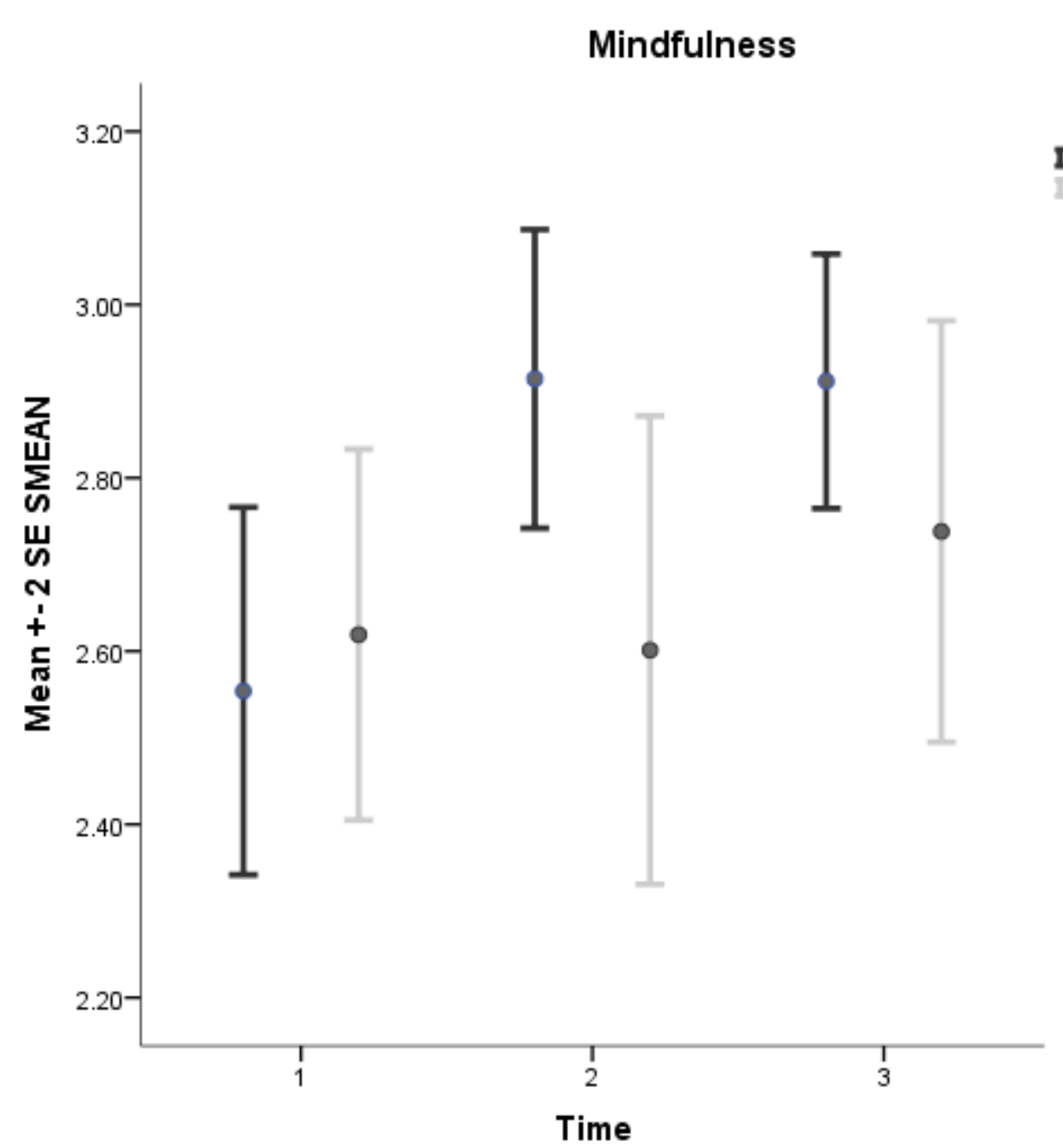

Figure 2. Effect of meditation on mindfulness skills. 
Emotional Intelligence

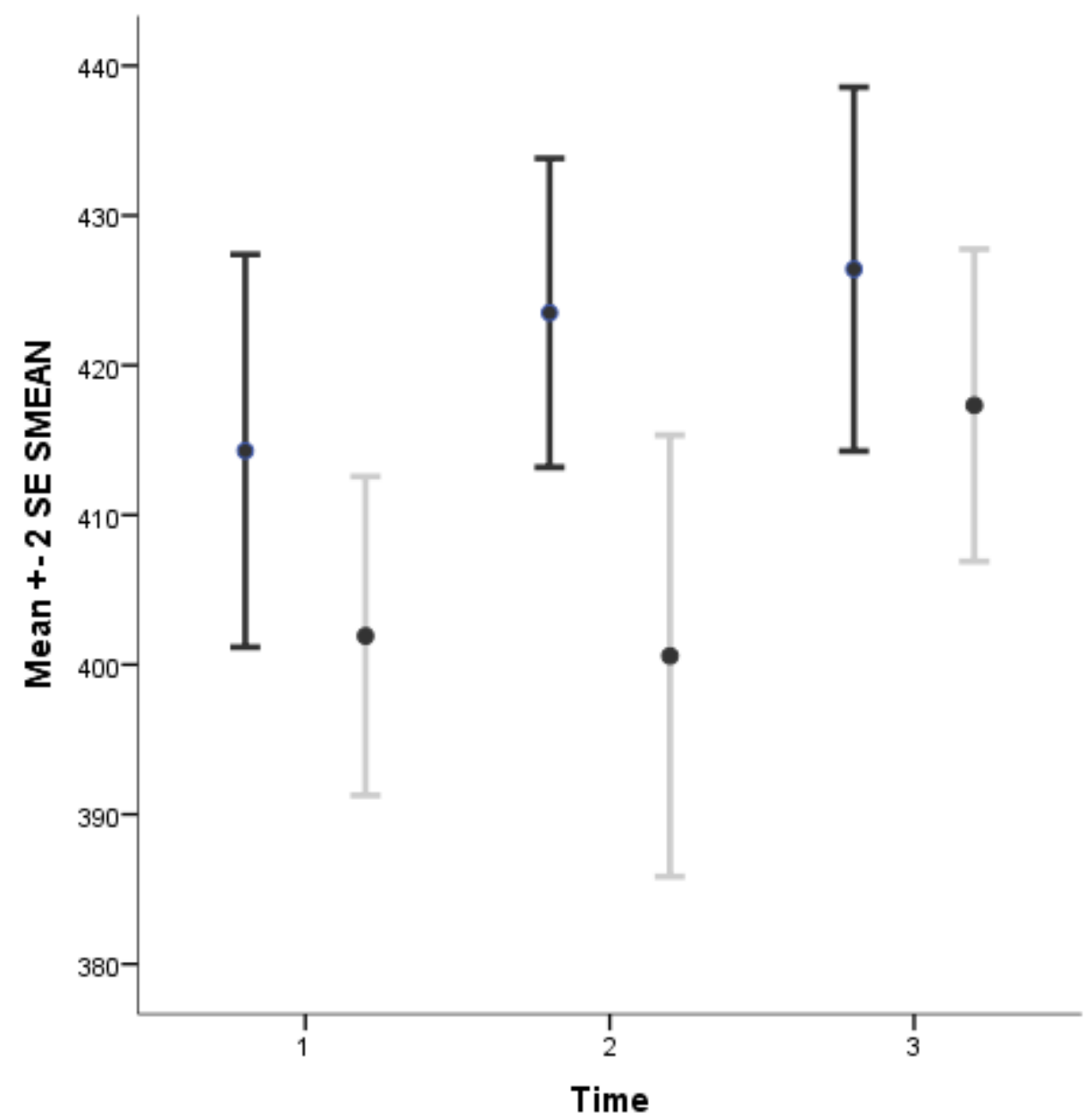

\section{Groups:}

I 1.Experimental/conrol 2.Control/experimental

Figure 3. Effect of meditation on emotional intelligence skills.

Job satisfaction. Job satisfaction did not differ between groups; $F(2,38)=.41, p=0.52$, with a small effect size of $\eta 2=.011$. It also did not differ over the study period; $F(2,38)=2.74, p=0.07$, with a small effect size of $\eta 2$ $=.067$.

Job stress-related presenteeism. There was no change in job stress-related presenteeism between groups; $F(2,38)$ $=2.54, p=0.119$, with a small effect size of $\eta 2=.063$. The main effect for time was also not significant; $F$ ( 2 , $38)=1.64, p=0.20$, with a small effect size of $\eta 2=.042$.

To test for the direct and interaction effects of presenteeism (Time 1) and mindfulness (Time 3) with perceived mental and physical health (Time 3), we used the PROCESS macro for SPSS (Hayes \& Rockwood, 2016) with bootstrapping $(N=5000)$. Both the physical health and mental health models support an interaction between the variables.

Table 1. Linear model of predictors of mental health

\begin{tabular}{lllll}
\hline & $B$ & SE $B$ & $t$ & $p$ \\
\hline Constant & $76.05(71.52-80.58)$ & 2.24 & 33.83 & 0.001 \\
Presenteeism & $-6.91(-13.14-0.69)$ & 3.08 & -2.24 & 0.03 \\
Mindfulness & $7.98(-3.03-18.99)$ & 5.46 & 1.46 & 0.15 \\
Presenteeism x mindfulness & $13.80(1.94-25.66)$ & 5.88 & 2.34 & 0.02 \\
\hline
\end{tabular}


Presenteeism and mindfulness interact to best explain the relationship with poor mental and physical health (Tables 1 and 2). The Johnson-Neyman significance regions indicate a strong relationship between those with higher presenteeism and reduced mental and physical health for those participants with lower mindfulness (mental health $<60$ th percentile and physical health $<73^{\text {rd }}$ percentile). Participants with mindfulness scores above these points did not show associations between presenteeism and perceived health. The findings suggest that higher mindfulness buffers the effect of presenteeism as these participants reported better mental and physical health irrespective of presenteeism at baseline.

Table 2. Linear model of predictors of physical health

\begin{tabular}{lllll}
\hline & $B$ & SE $B$ & $t$ & $P$ \\
\hline Constant & $80.55(74.50-86.60)$ & 2.99 & 26.88 & 0.001 \\
Presenteeism & $-4.81(-11.03-1.40)$ & 3.08 & -1.56 & 0.12 \\
Mindfulness & $1.00(-9.13-11.13)$ & 5.02 & 0.19 & 0.84 \\
Presenteeism x mindfulness & $-8.86(-15.98-1.75)$ & 3.52 & -2.51 & 0.01 \\
\hline
\end{tabular}

Note. $R^{2}=0.07, N=46$

Training evaluation. The mean score for the 14 closed questions on the Training Evaluation form was 4.67 of a possible 5 , suggesting high satisfaction with the training.

\subsection{Qualitative Analysis}

The application and impacts of the meditation intervention produced a range of responses from the participants. The preliminary codes organised relatively well into the three identifiable key themes; positive personal changes, good meditation training, and the company commitment and support. The theme of 'positive personal changes' included a subtheme of applying meditation learning to job role.

Positive personal changes were the strongest reported outcome of the meditation training and practice. The participants also mentioned the fact that they learned a valuable skill, expanded their knowledge, and enjoyed the process along the way. The theme spoke of the participants observing beneficial changes in themselves. Changes for some hinted at a new direction that their life would take:

- Meditation is and will now become a part of my daily routine

- Now I am more conscious about the things I can't control and don't stress about it

-I have become less reactive and more accepting

- I have meditation in my diary and don't schedule any meetings

Some participants commented on specific positive physiological changes that the training has produced:

-I am sleeping much better

- Feel better after meditating

- I feel 'gluggy' when I don't meditate on the weekends

- Ifeel a lot calmer

- Increased energy levels

- More alert

Others noted the beneficial impact of the course on others:

- I am more patient with my daughter

- Able to cope better with distractions at home.... and the family likes me for that

The theme of positive personal changes was closely associated with the subtheme of applying meditation learning to job role. Meditation during work hours enables the team to focus, declutter the mind, communicate better, and practice mindfulness:

- Able to 'scan' the situation better and make decisions a bit easier

- Better clarity and the ability to focus on goals

- Calmer and work through things better

- More energy to put into my work 
Good meditation training was directly related to the experience of the presenter and the effective training methods:

- Well described, good instructions, learned by doing

- We had an excellent teacher

- I liked physiological explanations and the effects on the body

- Course was great

- Four sessions in a row were good to create a habit and get into the swing

- Enjoyed the long meetings at the beginning

- Content was relevant and not attached to any belief system which is important; non-spiritual

Some highlighted the group experience as an important factor in learning and succeeding. Individuals enjoyed sharing knowledge with others and being part of a group:

- Learned from other people's experiences

- Sharing and participating together

Company commitment and support to promoting the health and well-being of team members in the workplace was evident throughout the study:

- Good having instructions during working hours

- Great to use work time for personal benefits

- Really nice for (company name) to prioritise our health and happiness at work

The findings from this study suggest that participants in the meditation intervention benefited in several ways. The participants reported improvement in both EI and mindfulness. Most importantly, the participants experienced positive personal changes in themselves. Many of these changes were translated into their job roles.

\section{Discussion}

The main purpose of this mixed methods study was to measure the impact of meditation on mindfulness skills, EI, job satisfaction, and job stress-related presenteeism in an Australian workplace. Our findings provided evidence that meditation training and participation enhance skills in mindfulness and EI. Our findings are commensurate with emotion regulation theory, as the participants, after mindfulness training, reported better understanding, awareness and expression of their emotions. The quantitative data analysis showed significant self-reported improvement in mindfulness, confirming that the level of mindfulness can be improved using ATMT meditation. This finding is consistent with past studies (Charoensukmongkola, 2014; Jha, Krompinger, \& Baime, 2007).

The findings also indicated that EI is a trainable skill and can be enhanced using mindfulness meditation. The results add support to the empirical investigations that have examined the relationship between EI and meditation (Chiesa et al., 2011). Individuals who are mindful are more adept at recognising how to behave appropriately in different situations. They detect the triggers of their emotions and therefore are able to predict and manage their emotions (Joseph \& Newman, 2010). The participants in the present investigation reported that the mindfulness and EI skills developed through meditation were applicable in the workplace, as well as in their personal lives.

There was no support for the second hypothesis that meditation training and participation leads to increased job-satisfaction and decreased stress-related presenteeism, which is contrary to some of the literature in this area (Levy, Wobbrock, Kaszniak, \& Ostergren, 2012; Shiba et al., 2015; Shonin et al., 2014). In the present study, however, the participating staff were already highly satisfied and displaying low levels of presenteeism and therefore unlikely to improve further with this short-term intervention due to a ceiling effect. Other explanations may be that the improvements in mindfulness and EI happened in advance of improvements in job satisfaction and stress-related presenteeism, the duration of data collection was not able to capture these changes, or the study lacked sufficient power to identify changes in these areas.

The findings do however, support the third hypothesis that mindfulness moderates the effects of stress-related presenteeism on health. The statistical approach allowed for an assessment of how participants with differing levels of mindfulness at the end of the program impacted the relationship between presenteeism and self-reported mental and physical health. Our findings suggest that high levels of mindfulness buffers the impact of presenteeism upon mental and physical health. For those with lower levels of mindfulness, the association was as anticipated, with high levels of presenteeism associated with reports of poorer physical and mental health. 
Our qualitative data suggest that participants in the meditation intervention benefited in several ways. Most importantly, the participants reported positive personal changes. Based on the participants' self-reported statements, many of these changes were translated into their job roles and putting more energy into their work, which is consistent with previous quantitative studies (Shiba et al., 2015; Shonin et al., 2014). This study has clear public health implications for relatively low cost, easy to implement health promoting activities at the workplace.

There are limitations of this study, which was planned to test the intervention and measurement tools. Further longitudinal research is required to track all meditation effects.

\subsection{Conclusions}

The current study explored the effects of meditation on EI, mindfulness, job satisfaction, and job stress-related presenteeism in an Australian corporate setting. The participants reported clear improvement in mindfulness and EI skills, with those with high levels of mindfulness potentially protected from the deleterious health effects of presenteeism.

The results of this study have important implications for workplaces. Given that clerical and administrative staff are traditionally hired for their technical expertise, greater mindfulness and emotional intelligence skills allow them to be more inspiring, engaging, and empathetic. These skills, together with the traditional skill set, comprise effective leaders, enable to better increase organisational effectiveness (Marques, 2013). Mindfulness is an essential tool of self-awareness and our findings show it has an important effect in buffering the harmful effects of stress-related presenteeism on employees' health. Developing workplace mindfulness training requires an ongoing, supporting infrastructure, consistent with a health promoting workplace.

\subsection{Recommendations for Further Research}

Further research is required to establish the direct and indirect impact of mindfulness, not only on health and wellbeing, but also on the employees' work performance. In addition, a study with a larger number of participants may determine whether the significant moderating effect of mindfulness on health outcomes of job stress-related presenteeism can be observed. Our findings demonstrate that mediation enhances mindfulness and EI; consequently, organisations can use meditation training and practice as a workplace intervention, especially for employees with higher emotional burdens, stress-related presenteeism or job demands.

\section{Acknoweledgments}

This research was a joint project between La Trobe University and Swisse Wellness Pty Ltd. and was funded in part by Swisse Wellness. The researchers acknowledge the support of Jonni Pollard, co-founder of 1 Giant Mind and the participants who took part in this research and kindly shared their time and experience.

\section{References}

Alexander, C. N., Swanson, G. C., Rainforth, M. V., Carlisle, T. W., Todd, C. C., \& Oates Jr., R. M. (1993). Effects of the Transcendental Meditation program on stress reduction, health, and employee development: A prospective study in two occupational settings. Anxiety, Stress \& Coping: An International Journal, 6(3), 245-262. https://doi.org/10.1080/10615809308248383

Baumeister, R. F., \& Heatherton, T. F. (1996). Self-regulation failure: an overview. Psychological Inquiry, 7(1), 1-15. https://doi.org/10.1207/s15327965pli0701_1

Charoensukmongkola, P. (2014). Benefits of Mindfulness Meditation on Emotional Intelligence, General Self-Efficacy, and Perceived Stress: Evidence from Thailand. Journal of Spirituality in Mental Health, 16(3), 171-192. https://doi.org/10.1080/19349637.2014.925364

Chiesa, A., Calati, R., \& Serretti, A. (2011). Does mindfulness training improve cognitive abilities? A systematic review of neuropsychological findings. Clin Psychol Rev, 31(3), 449-464. https://doi.org/10.1016/j.cpr.2010.11.003

Chu, L.-C. (2010). The benefits of meditation vis-à-vis emotional intelligence, perceived stress and negative mental health. Stress and Health, 26(2), 169-180. https://doi.org/10.1002/smi.1289

Colligan, T. W., \& Higgins, E. M. (2006). Workplace stress: Etiology and consequences. Journal of workplace behavioral health, 21(2), 89-97. https://doi.org/10.1300/J490v21n02_07

Gilbreath, B., \& Frew, E. J. (2008). The stress-related presenteeism scale [Measurement instrument]. Colorado State University, Hasan School of Business, Pueblo, CO.

Gilbreath, B., \& Karimi, L. (2012). Supervisor behavior and employee presenteeism. International Journal of Leadership Studies, 7(1), 114-131. 
Goodson, K. A. (2013). The benefits and implementation of an employee yoga program. In C. Smallwood \& L. B. Wade (Eds.), Job Stress and the Librarian: Coping Strategies from the Professionals. Jefferson, NC, USA: McFarland \& Company.

Goyal, M., Singh, S., Sibinga, E. M., Gould, N. F., Rowland-Seymour, A., Sharma, R., . . . Ranasinghe, P. D. (2014). Meditation Programs for Psychological Stress and Well-being: A Systematic Review and $\begin{array}{llll}\text { Meta-analysis. JAMA } & \text { Internal }\end{array}$ https://doi.org/10.1001/jamainternmed.2013.13018

Gross, J. (1998). The emerging field of emotion regulation: an integrative review. Psycholological Review of General Psychology, 2, 271-299. https://doi.org/10.1037/1089-2680.2.3.271

Laschinger, H. K. S., Cummings, G., Leiter, M., Wong, C., MacPhee, M., Ritchie, J., . . . Jeffs, L. (2016).Starting Out: A time-lagged study of new graduate nurses' transition to practice. International Journal of Nursing Studies, 57, 82-95. https://doi.org/10.1016/j.ijnurstu.2016.01.005

Hayes, A. F., \& Rockwood, N. J. (2016). Regression-based statistical mediation and moderation analysis in clinical research: Observations, recommendations, and implementation. Behaviour Research and Therapy, 98, 39-57.

Healthdirect. (2016). Meditation. Retrieved from https://www.healthdirect.gov.au/meditation

Herrman, H., Patrick, D. L., Diehr, P., Martin, M. L., Fleck, M., Simon, G. E., \& Buesching, D. P. (2002). Longitudinal investigation of depression outcomes in primary care in six countries: the LIDO study. Functional status, health service use and treatment of people with depressive symptoms. Psychological Medicine, 32, 889-902. https://doi.org/10.1017/S003329170200586X

Hoge, E. A., Guidos, B. M., Mete, M., E., B., Pollack, M. H., Simon, N. M., \& Dutton, M. A. (2017). Effects ofmindfulness meditation on occupational functioning and health care utilization in individuals with anxiety. Journal of Psychosomatic Research, 95, 7-11. https://doi.org/10.1016/j.jpsychores.2017.01.011

Ikin, J. F., Sim, M. R., Creamer, M. C., Forbes, A. B., McKenzie, D. P., Kelsall, H. L., . . Schwarz, H. (2004). War-related psychological stressors and risk of psychological disorders in Australian veterans of the 1991 Gulf War. British Journal of Psychiatry, 185, 116-126. https://doi.org/10.1192/bjp.185.2.116

Jha, A. P., Krompinger, J., \& Baime, M. J. (2007). Mindfulness training modifies subsystems of attention. Cognitive, Affective, \& Behavioral Neuroscience, 7(2), 109-119. https://doi.org/10.3758/CABN.7.2.109

Joseph, D. L., \& Newman, D. A. (2010). Emotional intelligence: An integrative meta-analysis and cascading model. Journal of Applied Psychology, 95(1), 54-78. https://doi.org/10.1037/a0017286

Karimi, L., Cheng, C., Bartram, T., Leggat, S. G., \& Sarkeshik, S. (2015). The effects of emotional intelligence and stress - related presenteeism on nurses' well - being. Asia Pacific Journal of Human Resources, 53(3), 296-310. https://doi.org/10.1111/1744-7941.12049

Karimi, L., Leggat, S., Donohue, L., Farrell, G., \& Couper, G. (2014). Emotional rescue: The role of emotional intelligence and emotional labour on wellbeing and job-stress among community nurses. Journal of Advanced Nursing, 70(1), 176-186. https://doi.org/10.1111/jan.12185

Khoury, B., Sharma, M., Rush, S. E., \& Fournier, C. (2015). Mindfulness-based stress reduction for healthy individuals: A meta-analysis. $J$ Psychosom Res, 78(6), 519-528. https://doi.org/10.1016/j.jpsychores.2015.03.009

Kulkarni, P. M., Janakiram, B., \& Kumar, D. N. S. (2009). Emotional Intelligence and Employee Performance as an Indicator for Promotion, a Study of Automobile Industry in the City of Belgaum, Karnataka, India. International Journal of Business and Management, 4(4), 161-170. https://doi.org/10.5539/ijbm.v4n4p161

Levy, D. M., Wobbrock, J. O., Kaszniak, A. W., \& Ostergren, M. (2012). Proceedings of Graphics Interface: The effects of mindfulness meditation training on multitasking in a high-stress information environment. Paper presented at the Canadian Information Processing Society Toronto, Ont., Canada.

Lutz, A., Slagter, H. A., Dunne, J. D., \& Davidson, R. J. (2008). Attention regulation and monitoring in meditation. Trends in Cognitive Sciences, 12(4), 163-169. https://doi.org/10.1016/j.tics.2008.01.005

Marques, J. (2013). Understanding the strength of gentleness: Soft-skilled leadership on the rise. Journal of Business Ethics, 116(1), 163-171. https://doi.org/10.1007/s10551-012-1471-7 
Medibank Private. (2008). The Cost of Workplace Stress in Australia Retrieved from http://www.medibank.com.au/client/documents/pdfs/the-cost-of-workplace-stress.pdf

National Institutes of Health. (2016). Meditation in Depth. Retrieved from https://nccih.nih.gov/health/meditation/overview.htm

Pascoe, M. C., \& Crewther, S. G. (2016). A Systematic Review of Randomised Control Trials Examining the Effects of Mindfulness on Stress and Anxious Symptomatology. In Anxiety Disorders. In Dover, DE, USA: SM Open Access eBook.

Schutte, N. S., \& Malouff, J. M. (2011). Emotional intelligence mediates the relationship between mindfulness and subjective well-being. Personality and Individual Differences, 50(7), 1116-1119. https://doi.org/10.1016/j.paid.2011.01.037

Seashore, S. E., Lawler, E. E., Mirvis, P., \& Cammann, C. (1982). Observing and measuring organizational change: A guide to field practice. New York: Wiley.

Sharma, M., \& Rush, S. E. (2014). Mindfulness-based stress reduction as a stress management intervention for healthy individuals: a systematic review. J Evid Based Complementary Altern Med, 19(4), 271-286. https://doi.org/10.1177/2156587214543143

Shiba, K., Nishimoto, M., Sugimoto, M., \& Ishikawa, Y. (2015). The Association between Meditation Practice and Job Performance: A Cross-Sectional Study. PLoS ONE, 10(5), e0128287. https://doi.org/10.1371/journal.pone.0128287

Shonin, E., Van Gordon, W., Dunn, T. J., Singh, N. N., \& Griffiths, M. D. (2014). Meditation Awareness Training (MAT) for Work-related Wellbeing and Job Performance: A Randomised Controlled Trial. International Journal of Mental Health and Addiction, 12(6), 806-823. https://doi.org/10.1007/s11469-014-9513-2

Slaski, M., \& Cartwright, S. (2002). Health, performance and emotional intelligence: an exploratory study of retail managers. Stress \& Health, 18(2), 63-68. https://doi.org/10.1002/smi.926

Statistical Solutions. (2015). Emotional Quotient Inventory (EQ-i) 2.0. Retrieved from http://www.statisticssolutions.com/emotional-quotient-inventory-eq-i-2-0/.

Tabachnick, B. G., \& Fidell, L. S. (2013). Cleaning up your act: Screening data prior to analysis. Using Multivariate Statistics, 6th Ed. (pp. 60-116). Boston, MA: Pearson Education.

Taylor, A. W., Wilson, D. H., Dal Grande, E., Ben-Tovim, D., Elzinga, R. H., Goldney, R. D., . . Kirke, K. (2000). Mental health status of the South Australian population. Australian and New Zealand Journal of Public Health, 24, 29-34. https://doi.org/10.1111/j.1467-842X.2000.tb00719.x

Travis, F., Haaga, D., Hagelin, J., Arenander, A., Tanner, M., \& Schneider, R. (2010). Self-referential awareness: Coherence, power, and eloreta patterns during eyes-closed rest, Transcendental Meditation and TM-sidhi practice. Journal of Cognitive Processing, 11(1), 21-30. https://doi.org/10.1007/s10339-009-0343-2

University of Wisconsin-Madison. (2008). Compassion Meditation Changes The Brain. ScienceDaily. Retrieved from http://www.sciencedaily.com/releases/2008/03/080326204236.htm.

Walach, H., Buchheld, N., Buttenmuller, V., Kleinknecht, N., \& Schmidt, S. (2006). Measuring Mindfulness--The Freiburg Mindfulness Inventory (FMI). Personality and Individual Differences, 40(8), 1543-1555. https://doi.org/10.1016/j.paid.2005.11.025

Ware, J. E., Kosinski, M., \& Keller, S. D. (1996). A 12-Item Short-Form Health Survey: Construction of Scales and Preliminary Tests of Reliability and Validity. Medical Care, 34(3), 220-233. https://doi.org/10.1097/00005650-199603000-00003

\section{Copyrights}

Copyright for this article is retained by the author(s), with first publication rights granted to the journal.

This is an open-access article distributed under the terms and conditions of the Creative Commons Attribution license (http://creativecommons.org/licenses/by/4.0/). 